\title{
Investigation of Gas Cooling Effect on the In Situ Heating Stage Inside Environmental TEM
}

\author{
Meng Li ${ }^{1}$, Degang Xie ${ }^{1}$, Xixiang Zhang ${ }^{2}$, Zhiwei Shan ${ }^{1}$ \\ 1. Center for Advancing Materials Performance from the Nanoscale (CAMP-Nano), State Key \\ Laboratory for Mechanical Behavior of Materials, Xi'an Jiaotong University, Xi' an 710049, China. \\ 2. King Abdullah University of Science \& Technology (KAUST), Division of Physical Science and \\ Engineering, Thuwal 23955-6900, Saudi Arabia
}

In-situ heating technique has been widely used in studies of temperature related material behaviors, such as phase transformations, solid/gas-solid reactions, microstructural changes, growth of nanostructures, sintering of catalysts, et al. A perfect heating stage for experiments inside TEM requires low spatial drifts and accurate temperature measurement during temperature rise and fall. Current commercial heating stages can be divided into three categories: conventional furnace heating, direct filament heating and MEMS based heating chips. In recent years, although the spatial resolution of current heating stages has been improved to atomic scale, accurate temperature control lags far behind, especially in case of gaseous environments. With the development of the environmental TEM in the past few years, increasing numbers of heating experiments are carried out in gas environments, especially in the field of catalysts. Gas introduction usually cools down the heated parts, and fluctuation of gas pressure and flow state also change the sample temperature dynamically. Therefore, accurate temperature measurement is critical to achieve precise temperature control in environmental TEM. However, most of the current insitu heating stages are designed without real-time temperature sensing, the temperature is only calculated from previous calibrations in vacuum. In this work, a home-made MEMS based in-situ heating stage with real-time temperature sensing and feed-back temperature control will be shown. With exquisite structural optimization, we also achieved so far the lowest thermal drift rate for in situ TEM imaging. Using this new device, the dynamic change of temperature with gas pressure are quantified systematically with several conventional gas species like $\mathrm{O}_{2}, \mathrm{~N}_{2}, \mathrm{H}_{2}$ and Air. Our presentation will introduce this new heating device and would provide a general reference for estimation of the actual sample temperature for device without temperature sensing in gas environments [1].

\section{References:}

[1] The authors acknowledge funding from Natural Science Foundation of China (NSFC) (51231005, 11132006 and 51321003). The authors thank Dr. Longqing Chen from Nanofabrication Core Lab, King Abdullah University of Science and Technology (KAUST) for the help in the MEMS fabrication. 

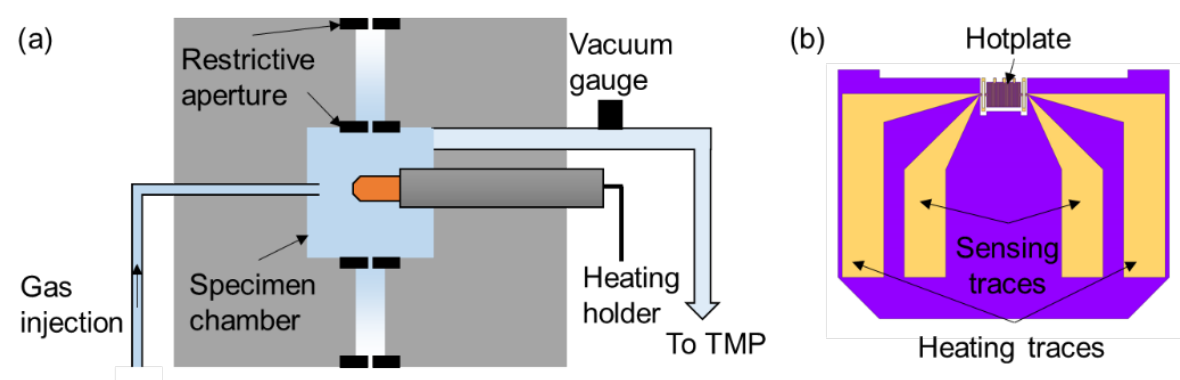

Figure 1. (a) Illustration of the experiment setup inside the environmental TEM. the heating chip is installed on the heating holder. The gas is injected into the specimen chamber using a gas injection nozzle, the gas pressure is measured by the vacuunm gauge. (b) Illustration of the home-made MEMS heating chip with both heating and temperature sensing availability. With structual optimazition, the temperature is uniformly distributed in the hotplate and thermally isolated with the rest parts to achieve accurate temeprature control. 